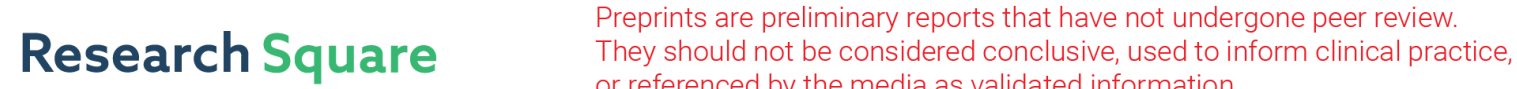 or referenced by the media as validated information. \\ A Comparative Study of Spiritual Needs of Thai Terminal III Cancer Patients
}

\section{Wasinee Wisesrith}

Chulalongkorn University

Pilaiporn Sukcharoen ( $\square$ pilaiporn.navynurse@gmail.com )

Suratthani Rajabhat University https://orcid.org/0000-0002-9352-9426

\section{Kanittha Sripinkaew}

Chulalongkorn University

\section{Research Article}

Keywords: Spiritual needs, Terminal ill cancer patients, Palliative care

Posted Date: April 1st, 2021

DOl: https://doi.org/10.21203/rs.3.rs-282663/v1

License: (1) This work is licensed under a Creative Commons Attribution 4.0 International License. Read Full License 


\section{Abstract}

Purpose Thai terminal ill cancer patients have spiritual distress and need spiritual care. They are in need of palliative care in the long term to alleviate suffering, achieve good spirituality, and to be ready to face with terminal ill conditions. This study aimed to investigate spiritual needs and compare differences in spiritual needs.

Methods A quantitative design was used for data collection and data analysis. The participants were Thai terminal ill cancer patients from Thai seven hospitals in northern, northeast, central, and southern regions; 322 participants were purposively selected. Instruments used was a spiritual needs scale. The instrument's validity was validated by 17 experts, a Delphi technique was performed, the Cronbach's alpha of 0.89 was found. Statistical analysis was F-test for one-way analysis of variance.

Results The overall spiritual needs of terminal ill cancer patients were at the moderate level. The highest mean was found in the 'prepare for death' dimension, followed by 'have meaning, values, and life purposes' and 'have opportunity to pursue most important things in life' dimensions consecutively and had different spiritual needs among status, number of family members, living with family, and spiritual anchor at the statistical significance level of .05.

Conclusion: The study results provide insight into spiritual needs of terminal ill cancer patients. That assists in improving the provision of holistic care to let the patients be happy at end-of-life phase.

\section{Introduction}

Currently, worldwide economic, social, and health conditions change rapidly. Also, there is a problem related to important health system in Thailand: There is continuing growth in the number of older adults, patients with chronic diseases, and cancer patients. Based on the National Cancer Institute of Thailand, 130,000 new cancer cases are found yearly. $39.96 \%$ of these cases were people aged 60 and over. In addition, cancer was the number one cause of death: there were $107.9 \%, 113.7 \%$, and $119.3 \%$ per 100,000 persons in 2014, 2015, and 2016 consecutively [1].

Moreover, patients are generally diagnosed as having incurable disease if cancer develops to the advanced stage. That affects the patients in all aspects of their lives, including physical, mental, psychosocial, and spiritual. Accordingly, terminal ill cancer patients are in need of palliative care in the long term to alleviate suffering resulted from the disease and burden of care the family carry [2-3]. Palliative care is an important care provided for terminal ill cancer patients - focuses on physical, mental, social, and spiritual care [4]. In addition, this science involves knowledge of holistic care built on an integration concerning healthcare individuals, community, and public policy aiming to alleviate suffering and promote quality of life of patients [5].

According to the aforementioned information, it is important that healthcare professionals becoming aware of the magnitude of holistic care provision for terminal ill cancer patients, specifically spiritual 
care. That is to serve care needs in all areas as well as to help the patients spend the rest of their lives with peace [3]. If any terminal ill cancer patient has developed the perception and achieve good spirituality, they will have positive experience of the illness and be ready to face with terminal ill conditions [5-6]. Therefore, registered nurses must have understanding of the spiritual needs of terminal ill cancer patients and families to provide efficient palliative care.

However, there is lack of specific health care systems for terminal patients in the context of the provision

of care for terminal ill cancer patients in Thai hospitals. There is no agency mainly in charge of palliative care provision, particularly in the dimensions of mental and spirituality of patients. The overall picture of care in Thailand is the provision of care in general, primarily focus on the physical dimension $[7,8]$. Accordingly, terminal ill cancer patients do not receive sufficient and efficient spiritual care $[9,10]$, the researcher aims to study spiritual needs of terminal ill cancer patients in the context of Thai culture to gain insight about their spiritual needs and compare differences in spiritual needs.

\section{Conceptual Framework}

The conceptual framework of this study based on the following the concept about palliative care which showed an important care provided for terminal ill cancer patients and holistic care to alleviate suffering of patients [4-5].

\section{Methods}

\section{Study design}

This study is a descriptive research. The participants were purposively selected based on the following criteria: Thai terminal ill cancer patients from Thai seven hospitals in northern, northeast, central, and southern regions who did not respond to the treatment, both male and female, admitted in the hospital, aged 40 and over, had the performance score of $40-70 \%$, had full consciousness, could communicate normally, and were willing to participate in this study. The sample size was determined applying rule of thumb: Number of multivariable was 10-20 times greater than the number of observable variables. Also, the research team added $15 \%$ of the sample size to increase the reliability; subsequently the sample size of 322 was derived.

\section{Research instruments}

\section{The spiritual needs scale}

The spiritual needs scale used in this study was developed applying a systematic literature review integrated with in-depth interview with terminal patients, family caregivers, and registered nurses with experience in providing care for patients. Content validity was validated by 17 experts, a Delphi technique was performed. The obtained median was 4.84 and interquartile range was 0.82 . For reliability testing, the Cronbach's alpha of 0.89 was found [5]. The instrument consisted of two sections: 
Section 1 Personal information: nine multiple-choice questions asking about age, marital status, number of family members, living with family, types of accommodation, religious belief, frequency of prayer, frequency of religious practice, and spiritual anchor.

Section 2 Spiritual needs scale: 41-item with 4-point rating scale, including eight dimensions: 1) have meaning, values, and life purposes ( 6 items); 2 ) gain love and encouragement from families and close persons ( 3 items); 3 ) review of life experience in the past (4 items); 4) have opportunity to pursue most important things in life (6 items); 5) prepare for death (8 items); 6 ) have opportunity to practice activities related to beliefs and faith was well as perform religious activities/rituals (6 items); 7 ) live in tranquil environment and atmosphere (4 items); and 8 ) needs to receive assistance in protecting human dignity (4 items)

\section{Data collection}

After getting a certificate of approval issued by the Institutional Review Board, the research team contacted head nurses and held a meeting with research assistants to clarify the objectives of the study and the measurement procedures. The research assistants were those with the following qualifications: hospital staff who were in charge of palliative care provision, had experience in the care, and had been trained for palliative care. Afterwards, questionnaires, consent forms, and participant information sheets were sent to the research assistants by post. The questionnaires were sent to measure spiritual needs of the participants; subsequently, the completed ones were sent back to the research team upon the due date.

\section{Data analysis}

1. Personal information was analyzed applying frequency and percentage.

2. Level of spiritual needs of the participants, both overall and by dimension, was analyzed applying mean and standard deviation

3. Comparing spiritual needs among those with varied age, marital status, number of family members, living with family, type of accommodation, religious belief, frequency of prayer, frequency of religious practice, and spiritual anchor applying an F-test for one-way analysis of variance. Pair comparison was performed when any mean difference was found.

\section{Results}

1. Personal information of 322 terminal ill cancer patients: age, marital status, number of family members, living with family, type of accommodation, religious belief, frequency of prayer, frequency of religious practice, and spiritual anchor. The majority of patients aged between 51 and 60 and between 61 and 70 (27.95\% and 27.95\% respectively). For marital status, the majority had married status (68\%). For number of family members, most patients had four family members (28\%). For living with family, the participants mostly lived with families (86.3\%). Type of accommodation 
mostly reported was stand-alone house (87.9\%). For religion, Buddhism (96\%) was most practiced. Praying daily (28.6\%) was most reported. For frequency of religious practice, perform sometimes (46.9\%) was mostly reported. For spiritual anchor, having spiritual anchor (68.9\%) was mostly reported.

2. The overall spiritual needs of terminal ill cancer patients was at the moderate level. The dimension with the highest mean score was 'prepare for death' which was at the high level. That was followed by two dimensions: 'have meaning, values, and life purposes' which was at the high level and 'have opportunity to pursue most important things in life' which was also at the high level. The dimension with the lowest mean score was 'live in tranquil environment and atmosphere' which was at the moderate level. These results are as shown in Table 1.

3. Terminal ill cancer patients with different marital status, number of family members, living with family, and spiritual anchor had different spiritual needs at the statistical significance level of .05. For those with different age, type of accommodation, religious belief, frequency of prayer, and frequency of religious practice; no difference in spiritual needs was found at the statistical significance level of .05. These results are as shown in Table 2.

To identify the difference in spiritual needs of terminal ill cancer patients with varied marital status, number of family members, living with family, and spiritual anchor; Least-Significant Difference method was performed. Results revealed: (1) Terminal ill cancer patients with married status had higher mean score of spiritual needs than those with widowed status. (2) Terminal ill cancer patients with 3-4 family members had higher mean score of spiritual needs than those with 1-2 family members. (3) Terminal ill cancer patients who lived with family had higher mean score of spiritual needs than those who lived alone. (4) Terminal ill cancer patients with 1 spiritual anchor had higher mean score of spiritual needs than those who had none. The results are as shown in Table 3-6

\section{Discussion}

The study results showed that the overall mean of spiritual needs of terminal ill cancer patients was at the moderate level. This can be explained that due to depending on medical devices to live because of incurable disease, terminal ill cancer patients are enduring physical, mental, social, and spiritual distress [11]. Moreover, these patients are unable to communicate their needs. Thus, the physical conditions of them are mainly cared for; meanwhile, other dimensions of their needs are neglected ${ }^{3}$. Registered nurses are the health care provider playing an important role in the delivery of holistic care for terminal ill cancer patients to alleviate suffering for the patients and their families [12-15]. If terminal ill cancer patients have religious beliefs and faith, positive attitudes towards life, and love from family; the patients view life as more meaningful and have peace of mind [16].

In addition, explain: if end-of-life needs are fulfilled and terminal ill cancer patients feel that hope exists, their quality of life will get better. Also, they will leave this world in peace. Accordingly, registered nurses should provide preparation for end-of-life phase for terminal ill cancer patients and their families [17-19]. 
That is to enable the patients to understand the illness, accept the conditions of the disease, and accept death [17,20-21]. Thus, spiritual care is an important approach that all registered nurses should keep in mind and provide it for terminal ill cancer patients [22-23].

The study results showed that terminal ill cancer patients with varied marital status, number of family members, living with family, and spiritual anchor differed in spiritual needs at the statistical significance level of .05. This can be explained that due to the conditions of incurable diseases, terminal ill cancer patients are enduring physical, mental, social, and spiritual distress. Their spiritual needs are varied in relation to beliefs, customs, tradition and culture, and religions and spiritual anchor that the patients and families have practiced [4]. The provision of holistic care for terminal ill cancer patients, especially the care related to the dimension of spirituality and beliefs of the patients and families is valuable and important in the area of palliative care for terminal ill cancer patients. That is to let the patient be happy at end-of-life phase and live this world with human dignity [6].

Considering the study results: (1) terminal ill cancer patients with married status had higher mean score of spiritual needs than those with widowed status. (2) terminal ill cancer patients with 3-4 family members had higher mean score of spiritual needs than those with 1-2 family members and 5 family members and over. (3) Terminal ill cancer patients who lived with family had higher mean score of spiritual needs than those who lived alone. It can be explained that terminal ill cancer patients feel happy when perceiving that they are valuable to others and families as well as able to perform behaviors that benefit others [24]. These patients are happy with being useful for others, perceive life as valuable, have self-esteem, satisfy with current situation in life, and have better spiritual well-being [24-25].

In addition, (4) Terminal ill cancer patients with 1 spiritual anchor had higher mean score of spiritual needs than those who had none. It can be explained that terminal ill cancer patients who have faith in religions will perform behaviors in line with the principles of their religions. The patients will have faith in rules, custom, tradition, values or culture of the society/sacred objects [3]. This will lead to living together in peace, find happiness in life, and have better spiritual well-being [5,24].

Moreover, spiritual needs of terminal ill cancer patients are varied depending on experience, culture, tradition, religion, and values. Therefore, having incurable disease is a major factor causing suffering in all areas, especially mental and spiritual distress. Some cases may be unable to accept death and end-oflife phase they are encountering, resulting in spiritual distress and no inner calm [5]. On the contrary, if terminal ill cancer patients develop the perception and achieve good spirituality; they would have positive experience related to their current illness, resulting in better quality of life [26].

In summary, religious beliefs and faith directly influence quality of life of terminal ill cancer patients. Each individual patient has different religious beliefs and needs [27-28]. When these needs are fulfilled, the patients will have peaceful life be able to accept illness' conditions and death better [26,29-30].

\section{Limitations of the study}


This study used data obtained only from Thai terminal ill cancer patients from Thai seven hospitals in northern, northeast, central, and southern regions who did not respond to the treatment. So, it may can't be generalized to other Thai terminal ill cancer patients who did respond to the treatment.

\section{Practical implications for nursing profession}

Nursing professional should investigate about spiritual need and other variables related to spiritual needs of Thai terminal ill cancer patients that encourages Thai terminal ill cancer patients to achieve holistic care in the long term to alleviate suffering and promote good quality of life of patients.

\section{Conclusion}

Terminal ill cancer patients are in need of palliative care in the long term to alleviate suffering resulted from the disease and burden of care the family carry. Health care professionals should have knowledge of patient care process and how to provide care for terminal ill cancer patients to alleviate the patients' suffering, specifically spiritual care. That is to serve care needs in all areas as well as to help the patients spend the rest of their lives with peace. The spirituality of terminal ill cancer patients varied according to perceptions, attitudes, experiences, and sociocultural conditions; especially when the patients facing suffering and incurable diseases. Health care professionals and registered nurses should be encouraged to continually assess spiritual needs of terminal ill cancer patients to accurately assess spiritual needs of each individual patient and provide specific care accordingly.

\section{Declarations}

- Funding: N/A

- Conflicts of interest/Competing interests: The authors declare that they have no conflict of interest.

- Availability of data and material: N/A

- Code availability: N/A

- Authors' contributions: N/A

- Ethics approval: The research objectives, protection of privacy of individuals, the right to participate to take part in the study with no effect on health care service provision. This study was approved by the Institutional Review Board of Chulalongkorn University (CU 1/59).

- Consent to participate: Informed consent was obtained from all the participants.

- Consent for publication: N/A

\section{References}

1. Health Administration Division (2012) Establishing a health service system development plan. http://bps.ops.moph.go.th/ moph/moph29Feb54/5. Accessed 13 December 2015 
2. World Health Organization (2012) WHO Definition of Palliative Care. http://www.who.int/cancer/palliative/definition/en/. Accessed 3 December 2015

3. Sukcharoen P, Sakunpong N, Sripa K (2020) Spiritual dimension in palliative care from the perspective of Thai palliative caregivers. International Journal of Palliative Nursing 26(2):70-74. http://doi.org/10.12968/ijpn.2020.26.2.

4. Chivantarak Foundation (2006) The five religion on take care to end of life patients. TNP Printing company, Bangkok

5. Wisesrith W, Soonthornchaiya R, Sukcharoen P (2019) Development and Preliminary Testing of The Spiritual Care Need Assessment Scale for End of Life Patients in Thai Cultural Context. Journal of Boromarajonani College of Nursing, Bangkok (35)5: 163-174

6. Daaleman PT, Reed D, Cohen W, Zimmerman S (2014) Development and Preliminary Testing of the Quality of Spiritual Care Scale. Journal of Pain and Symptom Management 47(4):793-800. http://doi.org/10.1016/j.jpainsymman.2013.06.004.

7. Poka K (2009) A study of professional nurse'perception on cultural congruent end of life care, tertiary hospitals under the jurisdiction of the ministry of public health. Master degree, Chulalongkorn University

8. Giovanni L (2012) End of Life Care in the United States: Current Reality and Future Promise A Policy Review. Nursing Economic 30(3):127-134. http://doi.org/22849010

9. Beccaro M, Aprile LP, Scaccabarozzi G, Cancian M, Costantini M (2013) Survey of Italian General Practitioners: Knowledge, Opinions, and activities of Palliative Care. Journal of Pain and Symptom Management 46(3):335-344. http://doi.org/10.1016/j.jpainsymman.2012.08.020.

10. Benito E, Oliver A, Galiana L, Barreto P, Pascual A, Gomis C, Barbero J (2013) Development and Validation of a New Tool for the Assessment and Spiritual Care of Palliative Care Patients. Journal of Pain and Symptom Management 47(6):1008-1018.

http://doi.org/10.1016/j.jpainsymman.2013.06.018.

11. Satawone D (2009) End of Life Care Improving Care of The Dying. Aksorn Sampan Press, Bangkok

12. Poor B, Poirrier G (2001) End of Life Nursing Care. Jones and Bartlett, London

13. Marrelli T (2005) Hospice and Palliative Care Handbook. Elsevier Mosby, Missouri

14. Taylor JE (2006) Prevalence and Associated Factors of Spiritual Needs Among Patients with Cancer and Family Caregivers. Oncology Nursing Forum 33(4):729-735. http://doi.org/11188/06.0NF.729735.

15. Astrow BA (2012) A Chinese Version of the Spiritual Needs Assessment for Patients Survey Instrument. Journal of Palliative Medicine 15(12):1297-1305. http://doi.org/1089/jpm.2012.0131.

16. Lin YL, Rau KM, Liu YH, Lin YH, Ying J, Kao CC (2015) Development and validation of the Chinese Version of Spiritual Interests Related IIIness Tool for patients with cancer in Taiwan. European Journal of Oncology Nursing 19:589-594. http://doi.org/10.1016/j.ejon.2015.03.005. 
17. Hermann PC (2006) Development and Testing of the Spiritual Needs Inventory for Patients Near the End of Life. Oncology nursing forum 33(4):737-744. http://doi.org/10.1188/06.0NF.737-744.

18. Vilalta A, Valls J, Porta J, Vinas J (2014) Evaluation of Spiritual Needs of Patients with Advanced Cancer in a Palliative Care Unit. Journal of palliative medicine 17(5):592-599. http://doi.org/10.1089/jpm.2013.0569.

19. Masso M, Allingham S, Johnson E, Pidgeon T, Yates P, Currow, D, Eagar K (2016) Palliative Care Problem Severity Score: Reliability and acceptability in a national study. Palliative Medicine 30(5): 479 - 485. http://doi.org/10.1177/0269216315613904.

20. Borneman T, Ferrell B, Puchalski C (2009) Evaluation of the FICA Tool for Spiritual Assessment. Journal of Pain and Symptom Management 40(2):163http://doi.org/10.1016/j.jpainsymman.2009.12.019.

21. Selman L, Harding R, Gysels M, Speck P, Higginson IJ (2011) The Measurement of Spirituality in Palliative Care and the Content of Tools Validated Cross-Culturally: A Systematic Review. Journal of Pain and Symptom Management 41(4):728-753. http://doi.org/10.1016/j.jpainsymman.2010.06.023.

22. Leow M, Chan M, Chan S (2014) Predictors of Change in Quality of Life of Family Caregivers of Patients Near the End of Life with Advanced Cancer. Cancer Nursing 37(5):391-399. http://doi.org/10.1097/NCC.0000000000000101.

23. Ando M, Morita T, Akechi T, Okamoto T (2010) Efficacy of Short-Term Life-Review Interviews on the Spiritual Well-Being of Terminally III Cancer Patients. Journal of Pain and Symptom Management 39(6):993-1002. http://doi.org/10.1016/j.jpainsymman.2009.11.320.

24. O'Brien (2017) Spirituality in nursing: Standing on holy ground. Jones \& Bartlett Learning, Massachusetts

25. Hungelmann J, Rossi EK, Klassen L, Stollenwerk R (1996) Focus on Spiritual Well-Being: Harmonious interconnectedness of mind-body-spirit-Use of the JAREL Spiritual Well-Being Scale: Assessment of spiritual well-being is essential to the health of individuals. Geriatric Nursing 17(6):262-266. http://doi.org/10.1016/s0197-4572(96)80238-2.

26. Boonyarat J, Uppanisakorn S (2014) The spirituality care in Intensive Patients and familys in Intensive Care Unit: Nursing Experiences. Princess of Narathiwat University Journal 4(1):1-13.

27. Shim E, Lee KS, Park JH (2010) Comprehensive needs assessment tool in cancer (CNAT): the development and validation. Support Care Cancer 19:1957- http://doi.org/10.1007/s00520-0101037-0.

28. Yong J, Kim J, Han SS, Puchalski CM (2008) Development and Validation of a Scale Assessing Spiritual Needs for Korean Patients with Cancer. journal of Palliative Care 24(4):240-287. http://doi.org/10.1177/082585970802400403.

29. Thongprateep T (2009) Nurse: being with the dying. V Print company, Bangkok 
30. Sukcharoen P, Sakunpong N, Sripa K (2020) Effectiveness of Transformative Learning on Spirituality in Palliative Care among Nursing Students: A Mixed Methods Study. The Journal of Behavioral Science 15(3):19-33. http://doi.org/10.12968/ijpn.2020.26.2.70.

\section{Tables}

\section{Table 1 level of spiritual needs.}

\begin{tabular}{|llll|}
\hline Spiritual needs of terminal ill cancer participants & $\overline{\mathbf{x}}$ & SD & Level \\
\hline 1. have meaning, values, and life purposes & 21.86 & 2.59 & High \\
\hline 2. gain love and encouragement from families and close persons & 11.11 & 1.40 & Medium \\
\hline 3. review of life experience in the past & 12.43 & 2.66 & Medium \\
\hline 4. have opportunity to pursue most important things in life & 21.40 & 2.83 & High \\
\hline 5. prepare for death & 28.85 & 3.49 & High \\
\hline $\begin{array}{l}\text { 6. have opportunity to practice activities related to beliefs and faith was } \\
\text { well as perform religious activities/rituals }\end{array}$ & 20.66 & 3.73 & High \\
\hline 7. live in tranquil environment and atmosphere & 14.67 & 1.81 & Medium \\
\hline 8. needs to receive assistance in protecting human dignity & 14.76 & 1.97 & Medium \\
\hline Overall & 18.21 & 2.56 & Medium \\
\hline
\end{tabular}

Table 2 Analysis of variance to compare means of spiritual needs. 


\begin{tabular}{|c|c|c|c|c|c|}
\hline Variance & SS & df & MS & F-test & P-value \\
\hline \multicolumn{6}{|l|}{ Age } \\
\hline Between group & 12393.52 & 51.00 & 243.01 & 1.09 & 0.32 \\
\hline Within group & 60117.26 & 270.00 & 222.66 & & \\
\hline Total & 72510.78 & 321.00 & & & \\
\hline \multicolumn{6}{|l|}{ Marital status } \\
\hline Between group & 2422.55 & 3.00 & 807.52 & 3.66 & $0.01 *$ \\
\hline Within group & 70088.24 & 318.00 & 220.40 & & \\
\hline Total & 72510.78 & 321.00 & & & \\
\hline \multicolumn{6}{|c|}{ Number of family members } \\
\hline Between group & 8313.01 & 8.00 & 1039.13 & 5.07 & $0.00 *$ \\
\hline Within group & 64197.77 & 313.00 & 205.10 & & \\
\hline Total & 72510.78 & 321.00 & & & \\
\hline \multicolumn{6}{|c|}{ Living with family } \\
\hline Between group & 1735.83 & 2.00 & 867.92 & 3.91 & $0.02^{*}$ \\
\hline Within group & 70774.95 & 319.00 & 221.87 & & \\
\hline Total & 72510.78 & 321.00 & & & \\
\hline \multicolumn{6}{|c|}{ Type of accommodation } \\
\hline Between group & 1088.92 & 6.00 & 181.49 & 0.80 & 0.57 \\
\hline Within group & 71421.87 & 315.00 & 226.74 & & \\
\hline Total & 72510.78 & 321.00 & & & \\
\hline \multicolumn{6}{|l|}{ Religious belief } \\
\hline Between group & 298.75 & 3.00 & 99.58 & 0.44 & 0.73 \\
\hline Within group & 72212.03 & 318.00 & 227.08 & & \\
\hline Total & 72510.78 & 321.00 & & & \\
\hline \multicolumn{6}{|c|}{ Frequency of prayer } \\
\hline Between group & 1492.54 & 4.00 & 373.14 & 1.67 & 0.16 \\
\hline Within group & 71018.24 & 317.00 & 224.03 & & \\
\hline Total & 72510.78 & 321.00 & & & \\
\hline
\end{tabular}




\begin{tabular}{|llllll|}
\hline Variance & SS & df & MS & F-test & P-value \\
\hline Frequency of religious practice & & & & & \\
\hline Between group & 1514.60 & 4.00 & 378.65 & 1.69 & 0.15 \\
\hline Within group & 70996.18 & 317.00 & 223.96 & & \\
\hline Total & 72510.78 & 321.00 & & & \\
\hline Spiritual anchor & & & & & \\
\hline Between group & 1828.68 & 2.00 & 914.34 & 4.13 & $0.02^{\star}$ \\
\hline Within group & 70682.10 & 319.00 & 221.57 & & \\
\hline Total & 72510.78 & 321.00 & & & \\
\hline
\end{tabular}

$* \mathrm{p}<.05$

Table 3 Mean comparisons of spiritual needs by marital status.

\begin{tabular}{|lllll|}
\hline Marital status & & Single & Married & Widowed \\
\cline { 2 - 5 } & $\overline{\mathrm{x}}$ & 141.64 & 147.58 & 142.01 \\
\hline Single & 141.64 & - & -5.93742 & -.36457 \\
\hline Married & 147.58 & 5.93742 & - & $5.57285^{\star}$ \\
\hline Widowed & 142.01 & .36457 & $-5.57285^{\star}$ & - \\
\hline${ }^{*} \mathrm{p}<.05$ & & & & \\
\hline
\end{tabular}

Table 4 Mean comparisons of spiritual needs by number of family members.

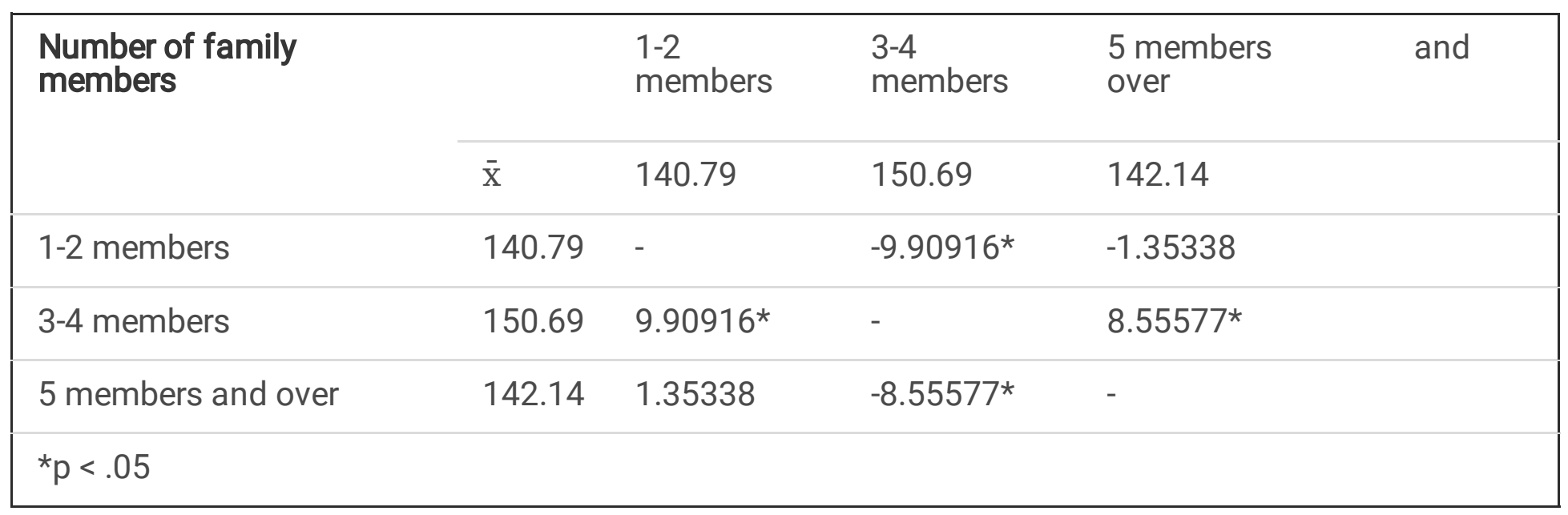


Table 5 Mean comparisons of spiritual needs by living with family.

\begin{tabular}{|c|c|c|c|c|}
\hline \multirow[t]{2}{*}{ Living with family } & & Live alone & Live with family & Live with friends \\
\hline & $\overline{\mathrm{x}}$ & 139.48 & 146.77 & 139.67 \\
\hline Live alone & 139.48 & - & $-7.28703^{*}$ & -.18391 \\
\hline Live with family & 146.77 & $7.28703^{\star}$ & - & 7.10312 \\
\hline Live with friends & 139.67 & .18391 & -7.10312 & - \\
\hline$\star p<.05$ & & & & \\
\hline
\end{tabular}

Table 6 Mean comparisons of spiritual needs by spiritual anchor.

\begin{tabular}{|c|c|c|c|c|}
\hline \multirow[t]{2}{*}{ Spiritual anchor } & & None & 1 spiritual anchor & 2 spiritual anchor and over \\
\hline & $\overline{\mathrm{x}}$ & 140.88 & 147.84 & 146.33 \\
\hline None & 140.88 & - & $-6.95936^{*}$ & -5.45035 \\
\hline 1 spiritual anchor & 147.84 & $6.95936^{\star}$ & & 1.50901 \\
\hline 2 spiritual anchor and over & 146.33 & 5.45035 & -1.50901 & \\
\hline
\end{tabular}

